\title{
Classification criteria for secondary Sjögren's syndrome. Current state of knowledge
}

\author{
Agata Sebastian ${ }^{1}$, Aleksandra Szachowicz ${ }^{2}$, Piotr Wiland ${ }^{1}$ \\ ${ }^{1}$ Department of Rheumatology and Internal Medicine, Wroclaw Medical University, Poland \\ ${ }^{2}$ East Kent Hospitals University NHS Foundation Trust, Ashford, UK
}

\begin{abstract}
Dryness of mucosa and eyes is the most recognizable symptom of Sjögren's syndrome (SS). Secondary SS (sSS) is diagnosed when symptoms of SS coexist with other systemic connective tissue disease. The sSS is often identified based on the symptoms of dryness in the presence of other connective tissue disease, making the diagnosis of sSS very subjective. The goal of this work was to summarize previously used and current criteria for the diagnosis of sSS. Classification criteria for sSS, which are universally accepted standards, do not exist. The diagnosis of sSS still depends on the experience of the investigator. Histopathological examination of minor salivary glands appears to be crucial for characterization of patients with sSS due to a specific picture of the disease and repeatability of the test.
\end{abstract}

Key words: autoimmune diseases, Sjögren's syndrome, classification criteria.

\section{Introduction}

Sjögren's syndrome (SS) belongs to a group of connective tissue diseases. Dryness of mucosa and eyes is the most common and most recognizable symptom of the disease. There are two forms of the disease: primary Sjögren's syndrome (pSS) and secondary Sjögren's syndrome (sSS).

Diagnosis of pSS is defined based on precisely defined classification criteria after excluding disorders that might imitate pSS, such as active hepatitis C, IgG4-dependent disease, or sarcoidosis, in the absence of other systemic connective tissue diseases [1]. On the other hand, sSS is diagnosed when symptoms of SS coexist with other systemic connective tissue disease. sSS is often identified based on the symptoms of dryness in the presence of other connective tissue disease, making the diagnosis of sSS very subjective.

The goal of this work was to summarize previously used and current criteria for the diagnosis of sSS.

\section{Methods}

The authors analyzed SCOPUS, MEDLINE and PubMed, EBESCO medical databases until April 2019 using the following key words: secondary Sjögren's syndrome, classification criteria.

\section{Classification}

There are no separate classification criteria for sSS. All previous guidelines only briefly refer to sSS criteria, which have not been created with sSS patients in mind and have never been validated in large groups of patients with sSS. Twelve different classification criteria for SS had been proposed by 2012 .

Classification criteria [1] (Table I) accepted by the American College of Rheumatology (ACR) and European League Against Rheumatism (EULAR) have been in effect since 2016. They constitute a consensus of the previous criteria [1-3]. These criteria are the most objective of all proposed to date due to exclusive use of objective methods, such as histopathological examination of labial salivary gland, Schirmer's test, staining of the eye with Rose Bengal, or assessment of unstimulated salivary flow.

In order to proceed with assessment for SS, a patient must fulfill the so-called preliminary criteria con-

Address for correspondence:

Agata Sebastian, Department of Rheumatology and Internal Medicine, Wroclaw Medical University, 213 Borowska St., 50-556 Wrocław,

Poland, e-mail: agatasebastian@vp.pl

Submitted: 3.09.2019; Accepted: 30.09.2019 
Table I. Current classification criteria for Sjögren's syndrome [based on 1]

\begin{tabular}{|lc|}
\hline Domain & $\begin{array}{c}\text { Weight } \\
\text { (points) }\end{array}$ \\
\hline Labial salivary gland biopsy showing focal lymphocytic sialadenitis and focus score $\geq 1 / 4 \mathrm{~mm}^{2}$ & 3 \\
\hline Anti-SSA/Ro antibodies & 3 \\
\hline Ocular staining score $\geq 5$ (or van Bijsterveld staining $\geq 4$ ) in at least one eye & 1 \\
\hline Schirmer's test $\leq 5 \mathrm{~mm} / 5 \mathrm{~min}$ in at least one eye & 1 \\
\hline Unstimulated total salivary excretion $\leq 0.1 \mathrm{ml} / \mathrm{min}$ & 1 \\
\hline
\end{tabular}

sisting of dryness of the eyes and mucus membranes or organ damage typical for pSS defined according to the EULAR Sjögren's syndrome disease activity index (ESSDAI). Symptoms of dryness typical for SS are assessed through questions regarding their duration and severity, such as those proposed by the American-European Consensus Group in 2002 (Table II) [3].

These criteria were the first to consider the importance of domains, indicating that specific symptoms from individual domains are not equivalent. Only the presence of specific antinuclear antibodies and histopathological examination of the labial salivary gland were considered characteristic for SS and each symptom was assigned 3 points.

In a publication referring to the 2016 diagnostic criteria, there is only one sentence that pertains to sSS, stating that these criteria may be used in patients with sSS. However, at the very beginning of the publication the researchers emphasize that these criteria have not yet been validated in patients with sSS and were created primarily for pSS patients.

The classification criteria from 1996 for SS diagnosis were based on observations of patients with both pSS and SSS, as well as patients with other connective tissue disorders and a group of patients with symptoms of dryness without accompanying autoimmune rheumatic disease [4].

Patients from 16 European and Israeli rheumatologic centers were analyzed. Patients with systemic connective tissue diseases were defined based on criteria applicable at the time [5-9]. Division into pSS and sSS was based exclusively on the opinion of experts participating in the study. sSS was reported by researchers as secondary to systemic lupus erythematosus (22\%), rheumatoid arthritis $(53 \%)$, or scleroderma (14\%). None of those centers reported sSS in patients with polymyositis, dermatomyositis or mixed connective tissue disease.

It was established that patients diagnosed with other systemic connective tissue diseases as well as those with eye or mouth dryness (Table II) and two of the following characteristics - positive Schirmer's test or staining with Rose Bengal confirming dry eye syndrome, focus score $\geq 1$ in histopathological examination of the labial salivary gland, or involvement of salivary glands confirmed with scintigraphy, sialography, or unstimulated salivary excretion test - fulfilled the diagnostic criteria for sSS.

These criteria did not take into consideration the presence of antibodies specific to SSA or SSB antigen due to their possible presence in the course of other connective tissue diseases. Specificity of the proposed criteria for sSS in the study group (278 patients) was $91.8 \%$ and sensitivity $97.3 \%$. These were the first criteria to abandon the division of SS into a definite and probable disease.

According to re-defined 2002 SS classification criteria, the diagnosis of sSS can be made in patients with another coexisting connective tissue disease, presence of domain 1 or 2 symptoms (ocular or oral, Table II), and positive results of two of the remaining studies: positive Schirmer's test or Rose Bengal staining confirming dry eye syndrome, focus score $\geq 1$ in histopathological examination of the minor salivary gland, or involvement of salivary glands confirmed with scintigraphy, sialography, or the unstimulated salivary flow test. Again, it was agreed that antibodies specific to SSA and SSB antigens cannot be considered as a marker for sSS.

In their publication the researchers did not indicate that tests assessing excretion of saliva (unstimulated salivary flow) or tears (Schirmer's test) should not be taken into consideration among patients after the age of 60 [3], as described in the 1996 SS criteria [4].

Subsequently, in 2012 Shiboski et al. [2] published the Sjögren's International Collaborative Clinical Alliance (SICCA) criteria. As indicated, these criteria were not created for patients with sSS, as those patients constituted a minority (6\%) of patients in the studied group.

The 2012 and 2016 criteria do not require symptoms of mucosal or eye dryness, allowing for earlier diagnosis of SS before development of fully symptomatic, typical disease. Table II presents a summary of classification criteria and their domains over the years 1996-2016.

\section{Dryness}

As many as $20 \%$ of patients suffering from rheumatoid arthritis report subjective symptoms of dryness [10]. However, only $5 \%$ of them fulfill the sSS criteria. Anti- 
Table II. Domains included in the classification criteria created since 1996 [based on 1-4]

\begin{tabular}{|c|c|c|c|c|c|}
\hline Domain & Classification criteria & 1996 & 2002 & 2012 & 2016 \\
\hline 1 - ocular & $\begin{array}{l}\text { Have you had daily, persistent, troublesome dry eyes } \\
\text { for more than } 3 \text { months? }\end{array}$ & + & + & & \\
\hline 1 - ocular & $\begin{array}{l}\text { Do you have a recurrent sensation of sand or gravel } \\
\text { in the eyes? }\end{array}$ & + & + & & \\
\hline 1 - ocular & $\begin{array}{l}\text { Do you use tear substitutes more than three times } \\
\text { a day? }\end{array}$ & + & + & & \\
\hline 2 -oral & $\begin{array}{l}\text { Have you had a daily feeling of dry mouth for more than } \\
3 \text { months? }\end{array}$ & + & + & & \\
\hline 2 - oral & $\begin{array}{l}\text { Have you had recurrently or persistently swollen } \\
\text { salivary glands as an adult? }\end{array}$ & + & + & & \\
\hline 2 -oral & $\begin{array}{l}\text { Do you frequently drink liquids to aid in swallowing } \\
\text { dry food? }\end{array}$ & + & + & & \\
\hline 3 - ocular signs & Schirmer test $\leq 5 \mathrm{~mm}$ in 5 minutes & + & + & & $\begin{array}{l}+ \text { in at least } \\
1 \text { eye }\end{array}$ \\
\hline 3 - ocular signs & $\begin{array}{l}\text { Rose Bengal score ( } \geq 4 \text { according to van Bijsterveld's } \\
\text { scoring system) }\end{array}$ & + & + & & $\begin{array}{l}+ \text { in at least } \\
1 \text { eye }\end{array}$ \\
\hline 3 - ocular signs & Ocular staining score $\geq 3$ & & & + & \\
\hline 3 - ocular signs & Ocular staining score $\geq 5$ & & & & $\begin{array}{l}+ \text { in at least } \\
1 \text { eye }\end{array}$ \\
\hline 4 - histopathology & Focus score $\geq 1$ in minor salivary gland biopsy & + & + & + & + \\
\hline 5 - salivary gland involvement & Salivary scintigraphy & + & + & & \\
\hline 5 - salivary gland involvement & Parotid sialography & + & + & & \\
\hline 5 - salivary gland involvement & Unstimulated salivary secretion $\leq 0.1 \mathrm{ml} / \mathrm{min}$ & + & + & & + \\
\hline 6 -immunological & anti-SSA antibodies & + & + & + & + \\
\hline 6 -immunological & anti-SSB antibodies & + & + & + & \\
\hline 6-immunological & antinuclear antibody (ANA) and rheumatoid factor (RF) & & & + & \\
\hline
\end{tabular}

gens against SSA or SSB rarely occurred in this group of patients with sSS.

It should be remembered that among patients with systemic sclerosis who develop xerostomia, this symptom is usually caused by fibrosis of salivary glands in the course of primary disease, not by their dysfunction. Interestingly, patients suffering from limited systemic sclerosis presented with labial salivary gland infiltration with mononuclear cells in as many as $60 \%$ of patients.

Major salivary gland enlargement occurs in 25-66\% of patients with pSS [11]. It is not, however, a common picture of sSS. Once again, this points to the differences between these groups of patients. Hence, there is a question whether PSS and SSS truly represent two different forms of the same disease. Or maybe they constitute two separate disorders?

Recently Mavragani et al. [12] proposed that the terms primary and secondary SS should be replaced by a more descriptive terminology: SS when the disease is expressed as an entity alone or SS associated with systemic or organ-specific autoimmune diseases, provided that in all cases, the recently published set of criteria for SS are fulfilled. So the term secondary no longer exists, and the presence of an underlying autoimmune disease does not exclude the classification of primary SS, once the proposed criteria are fulfilled and the notion of sSS should be currently referred to as polyautoimmunity (i.e., 2 or more autoimmune diseases in the same individual) [12].

\section{Antibodies}

Anti-SSA antibodies are not only present in SS. They can also be found in rheumatoid arthritis, lupus erythematosus, scleroderma and myositis [13-15], making them non-specific for sSS. Thus, their application in classification of sSS indicates a possibility of sSS, but does not confirm it.

Taking into consideration previously proposed and currently binding criteria for sSS, histopathological examination of the salivary gland is an objective test for the diagnosis of sSS. Evidently, objective tests assessing the amount of excreted saliva and tears also constitute the basis for the diagnosis of sSS, although it should be remembered that isolated test abnormalities may result from many different causes of eye and mouth dry- 
ness $[16,17]$ and tests should be conducted according to the protocols proposed by Whitcher, Bijsterveld and Navazesh [18-21].

Summarizing the classification criteria for sSS, it should be emphasized that we are not currently in possession of universally accepted standards. The most recently proposed criteria require validation in patients with sSS.

The diagnosis of sSS still depends on the experience of the investigator. However, sSS criteria are necessary for identifying groups of patients with sSS in order to determine the differences in the pathomechanism and clinical symptoms of both forms of SS as well as the impact of sSS on the course of primary disease, e.g. rheumatoid arthritis.

It should also be noted that the diagnostic criteria of sSS include histopathological examination of minor salivary glands, which appears to be crucial for characterization of patients with sSS due to the specific picture of the disease and repeatability of the test. Histopathological assessment should be conducted according to the standards implemented for SS [22].

Furthermore, an existing diagnosis can be modified after a change in disease classification criteria. This has happened particularly in SS. This fact should be taken into account when comparing groups of patients classified on the basis of different sets of criteria, and analyzing individual patients during a longer follow-up period [23].

\section{Conclusions}

In conclusion, we suggest the need for labial salivary gland biopsy in all cases in which we suspect sSS to confirm the diagnosis.

The authors declare no conflict of interest.

\section{References}

1. Shiboski CH, Shiboski SC, Seror R, et al. 2016 ACR-EULAR Classification Criteria for primary Sjögren's Syndrome: A Consensus and Data-Driven Methodology Involving Three International Patient Cohorts. Arthritis Rheumatol 2017; 69: 35-45.

2. Shiboski SC, Shiboski CH, Criswell L, et al. American College of Rheumatology Classification Criteria for Sjögren's Syndrome: A Data-Driven, Expert Consensus Approach in the SICCA Cohort. Arthritis Care Res (Hoboken) 2012; 64: 475-487.

3. Vitali C, Bombardieri S, Jonsson R, et al. Classification criteria for Sjögren's syndrome: a revised version of the European criteria proposed by the American-European Consensus Group. Ann Rheum Dis 2002; 61: 554-558.

4. Vitali C, Bombardieri S, Moutsopoulos HM, et al. Assessment of the European classification criteria for Sjogren's syndrome in a series of clinically defined cases: results of a prospective multicenter study. The European Study Group on Diagnostic Criteria for Sjögren's Syndrome. Ann Rheum Dis1996; 55: 116-121.
5. Arnett FC, Edworthy SM, Bloch DA, et al. The American Rheumatism Association 1987 revised criteria for the classification of rheumatoid arthritis. Arthritis Rheum 1988; 31: 315-324.

6. Tan EM, Cohen AS, Fries JF, et al. The 1982 revised criteria for the classification of systemic lupus erythematosus. Arthritis Rheum 1982; 25: 1271-1277.

7. Preliminary criteria for the classification of systemic sclerosis (scleroderma). Subcommittee for scleroderma criteria of the American Rheumatism Association Diagnostic and Therapeutic Criteria Committee. Arthritis Rheum 1980; 23: 581-590.

8. Sharp GC, Irvin WS, Tan EM, et al. Mixed connective tissue disease - an apparently distinct rheumatic disease syndrome associated with a specific antibody to an extractable nuclear antigen (ENA). Am J Med 1972; 52: 148-159.

9. Bohan A, Peter JB. Polymyositis and dermatomyositis (first of two parts). N Engl Med 1975; 292: 344-347.

10. Manoussakis MN, Moutsopoulos HM. Sjögren's syndrome: autoimmune epithelitis. Baillieres Best Pract Res Clin Rheumatol 2000; 14: 73-95.

11. Manoussakis MN, Moutsopoulos HM. Update knowledge of dry mouth - A guideline for dentists. Adv Intern Med. 2001; 47:191-217.

12. Mavragani CP, Moutsopoulos HM. Primary versus Secondary Sjögren Syndrome: Is It Time To Reconsider These Terms? J Rheumatol 2019; 46: 665-666.

13. Tipu HN, Bashir MM. Determination of Specificity and Pattern of Antinuclear Antibodies (ANA) in Systemic Rheumatic Disease Patients Positive for ANA Testing. I Coll Physicians Surg Pak 2018; 28: $40-43$

14. Sánchez-Montalvá A, Fernández-Luque A, Simeón CP, et al. Anti-SSA/Ro52 autoantibodies in scleroderma: results of an observational, cross-sectional study. Clin Exp Rheumatol 2014; 32 (Suppl 86): 177-182.

15. Defendenti C, Atzeni F, Spina MF, et al. Clinical and laboratory aspects of Ro/SSA-52 autoantibodies. Autoimmun Rev 2011; 10: 150-154.

16. Hashemi H, Khabazkhoob M, Kheirkhah A, et al. Prevalence of dry eye syndrome in an adult population. Clin Exp Ophthalmol 2014; 42: 242-248.

17. Alsakran Altamimi M. Update knowledge of dry mouth- A guideline for dentists. Afr Health Sci 2014; 14: 736-742.

18. Whitcher JP, Shiboski CH, Shiboski SC, et al. A simplified quantitative method for assessing keratoconjunctivitis sicca from the Sjogren's Syndrome International Registry. Am J Ophthalmol 2010; 149: 405-415.

19. Sebastian A, Markuszewska A, Markuszewski B, et al. Enhanced ocular staining score in patients with primary Sjögren's syndrome. Klin Oczna 2014; 116: 205-209.

20. Van Bijsterveld OP. Diagnostic tests in the Sicca syndrome. Arch Ophthalmol 1969: 82: 10-14.

21. Navazesh M, Kumar SK. Measuring salivary flow: challenges and opportunities. J Am Dent Assoc 2008: 139: 35-40.

22. Fisher BA, Jonsson R, Daniels T, et al. Standardisation of labial salivary gland histopathology in clinical trials in primary Sjögren's syndrome. Ann Rheum Dis 2017; 76: 1161-1168.

23. Wiland P. Panta rhei in diagnosing rheumatic diseases. Reumatologia 2019; 57: 127-128. 